\title{
Calculated Enhancement for Intracavity Spectroscopy with a Single-Mode Laser
}

\author{
H. J. KIMBLE
}

\begin{abstract}
A semiclassical description of the laser is employed to calculate the change in laser intensity due to the small perturbation of an absorbing medium within the laser cavity. The laser is assumed to be operating in a single-mode near its threshold of oscillation. An expression is derived for the enhancement in detection sensitivity of laser intracavity absorption measurements over conventional absorption measurements and estimates of detection sensitivity are given.
\end{abstract}

\section{INTRODUCTION}

$\mathrm{T}$ HE sensitivity of absorption measurements is greatly enhanced by the method of laser intracavity absorption spectroscopy in which an absorption cell is placed inside a laser cavity. Since the first demonstration of this enhancement by Pakhomycheva et al. [1] and by Peterson et al. [2]-[4], the technique has been successfully utilized in numerous experiments, including measurements of trace gas concentrations [5], free radical reaction rates [6], [7], and forbidden optical transitions [8]. Enhancements in sensitivity over conventional single-pass absorption measurements as high as $10^{5}$ have been reported [9].

A number of theoretical treatments of intracavity absorption spectroscopy appear in the literature [9]-[13] and are based on rate equation models for the laser plus absorber system. While these treatments provide adequate descriptions for laser operation above threshold, they are not applicable as the threshold of the laser oscillation is approached since they do not properly describe the effect of spontaneous emission.

In this paper, we investigate the near threshold behavior of a single-mode, $\mathrm{CW}$ laser with an intracavity absorber. The treatment is based on a simple extension of the well-known semiclassical theory by Risken [14] and deals with the case in which the absorber represents a small perturbation to the operation of the laser. Above threshold, our result for the intracavity enhancement agrees with the usual rate-equation approach [9]-[13], but very near threshold it is altered by the contribution from spontaneous emission. While our attention is restricted to the case of a single-mode laser, we demonstrate that the sensitivity enhancement for single-mode operation near threshold can be comparable to that achieved with intracavity absorption in multimode lasers operated far above threshold. Intracavity spectroscopy with a single-mode laser has the potentially attractive feature that the absorption spectrum of the intracavity absorber can be probed with high frequency resolution, unlike the case for multimode operation.

Manuscript received July 5, 1979; revised November 2, 1979.

The author was with the Physics Department, General Motors Research Laboratories, Warren, MI 48090. He is now with the Department of Physics, University of Texas at Austin, Austin, TX 78712.

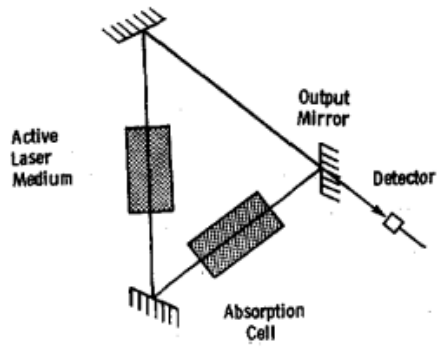

Fig. 1. Illustration of a laser cavity with an intracavity absorption cell.

We begin in Section II with a simple model to calculate the enhancement in intracavity spectroscopy. In Section III we extend the treatment to include the fluctuations due to spontaneous emission following Risken [14], and in Section IV we derive an expression for the enhancement expected near threshold.

\section{Intracavity Enhancement Far Above Threshold}

An absorber placed inside a laser cavity, as shown in Fig. 1, and with an atomic or molecular transition resonant with the laser frequency alters the laser intensity in a manner that can be understood as follows. In the steady state far above the threshold for laser operation, the intracavity laser intensity is determined by the requirement that the gain $G$ equal the loss $l$ for one roundtrip through the cavity [15]. Since the gain of a homogeneously broadened laser is a function of the internal intensity $I$,

$$
G=\frac{G_{0}}{1+B I}
$$

where $G_{0}$ is the small-signal gain and $B$ is the saturation parameter for the laser, we find upon setting $G=l$ and solving for $I$

$$
I=\frac{I}{B}\left(\frac{G_{0}}{l}-1\right)
$$

A small change $d l$ in the loss $l$ due to the insertion of an absorbing medium into the cavity produces a corresponding change in laser intensity $I$ given by

$$
d I=-\frac{G_{0}}{B l^{2}} d l
$$

so that

$$
\frac{d I}{I}=-d l\left\{\frac{n}{l(n-1)}\right\}
$$


with $n \equiv G_{0} / l$. If the same absorber were placed external to the cavity, the fractional change in intensity $d I / I$ would be simply - $d l$, which leads us to associate with the intracavity absorption an enhancement $\xi$

$$
\xi \equiv \frac{d I}{I} /-d l
$$

which from (3) is seen to be

$$
\xi=\frac{n}{l(n-1)} \text {. }
$$

An expression of this form is well known, both in the context of single-mode [12], [16] and multimode [9], [10] laser operation. It expresses the enhancement in terms of the working point of the laser $n$ and in terms of the multipass nature of an intracavity absorption cell, since the cavity loss $l$ may be expressed in terms of the reflectivity $R$ of the output coupling mirror

$$
l \simeq(1-R)
$$

for small distributed and diffraction losses within the cavity.

In the region of the laser threshold, $n$ approaches unity and the enhancement $\xi$ as given by (5) becomes arbitrarily large. If we are to correctly account for the laser behavior near threshold, we must adopt an approach that includes the effect of spontaneous emission. Toward this end we present in Section III an extension of the well-known semiclassical theory of the laser. Those readers uninterested in the details of this extension can go directly to Section IV for a calculation of the intracavity enhancement.

\section{LASER MODEL}

We wish to derive an equation for the laser intensity that includes the effect of an intracavity absorber as well as spontaneous emission. For the model of the laser we follow the semiclassical treatment by Risken [14] and consider a ring laser supporting a single polarized traveling wave interacting with an active laser medium of $N$ atoms, each represented by a two-level system with energy eigenstates denoted by $|1\rangle_{L}$ and $|2\rangle_{L}$ and separated by an energy $\left(E_{2}-E_{1}\right)=\hbar \omega_{0}$. In addition, the laser cavity is assumed to contain an absorbing material comprised of $N_{A}$ atoms described by the eigenstates $|1\rangle_{A}$ and $|2\rangle_{A}$ with the same transition frequency $\omega_{0}$. Both laser and absorber transitions are taken to be homogeneously broadened, and both groups of atoms interact via an electric dipole interaction with an electric field $E(z, t)$ propagating along the laser axis $z$, with polarization in the $x$ direction, and represented by [14]

$$
E(z, t)=\sqrt{\frac{\hbar \omega_{0}}{2 \epsilon_{0} V}}\left\{b(t) e^{-i \omega_{0}(t-z / c)}+\text { c.c. }\right\}
$$

where $V$ is the cavity volume. The amplitude $b(t)$ of the electric field is assumed to be a slowly varying function of time, such that $\dot{b} \ll \omega_{0} b$, and the normalization in (6) is chosen so that $b^{*} b$ gives the intensity of the electric field in terms of a photon number.

The Hamiltonian $H$ for an atom in the cavity interacting with the field $E$ is of the form

$$
H=H_{0}-e x E
$$

where $H_{0}$ describes the atom in the absence of the field and $(-e x E)$ represents the coupling of the atom to the field through the electric dipole interaction. The equations of motion for the elements $\rho_{i j} \equiv\left\langle i|\rho|_{j}\right\rangle$ of the density operator $\rho$ describing the $\mu$ th atom of the laser medium or the $\nu$ th atom of the absorbing medium are found from the relation

$$
\dot{\rho}=\frac{i}{\hbar}[\rho, H]
$$

and are given by

$$
\begin{aligned}
& \dot{\rho}_{12}^{(\mu)}=i \omega_{0} \rho_{12}^{(\mu)}+\frac{i e}{\hbar} x_{12} E\left(\rho_{22}^{(\mu)}-\rho_{11}^{(\mu)}\right)-\gamma_{2} \rho_{12}^{(\mu)} \\
& \dot{\rho}_{22}^{(\mu)}-\dot{\rho}_{11}^{(\mu)}=\frac{2 i}{\hbar} e x_{12} E\left(\rho_{12}^{(\mu)}-\rho_{21}^{(\mu)}\right) \\
& +\gamma_{1}\left[\frac{\sigma_{0}}{N}-\left(\rho_{22}^{(\mu)}-\rho_{11}^{(\mu)}\right)\right] \\
& \dot{\rho}_{12}^{(\nu)}=i \omega_{0} \rho_{12}^{(\nu)}+\frac{i e}{\hbar} x_{12}^{\prime} E\left(\rho_{22}^{(\nu)}-\rho_{11}^{(\nu)}\right)-\gamma_{2}^{\prime} \rho_{12}^{(\nu)} \\
& \dot{\rho}_{22}^{(v)}-\dot{\rho}_{11}^{(\nu)}=\frac{2 i}{\hbar} e x_{12}^{\prime} E\left(\rho_{12}^{(v)}-\rho_{21}^{(v)}\right)-\gamma_{1}^{\prime}\left[\rho_{22}^{(\nu)}-\rho_{11}^{(v)}+1\right]
\end{aligned}
$$

where $x_{12} \equiv\langle 1|x| 2\rangle_{T}=\langle 2|x| 1\rangle_{J^{\prime}}$ and $x_{12}^{\prime} \equiv\langle 1|x| 2\rangle_{A}=$ $\langle 2|x| 1\rangle_{A}$. As in Risken [14], (9) and (10) have been supplemented by the terms $\gamma_{2} \rho_{12}$, which accounts for decay of the off-diagonal elements of the density operator, $\gamma_{1}\left(\rho_{22}-\rho_{11}\right)$, which represents decay of the atomic population difference, and $\left(\gamma_{1} \sigma_{0} / N\right)$, which describes the pumping process producing a population inversion. Similar terms denoted by a prime have been added to (11) and (12) to describe the absorbing medium. In (12), the inversion is chosen so that in the absence of a field all absorber atoms are in the ground state.

The laser and absorber atoms interact through the field $E$, which is in turn determined by the polarization produced by these atoms by way of the wave equation

$$
\frac{\partial^{2} E}{\partial t^{2}}+2 K \frac{\partial E}{\partial t}-c^{2} \frac{\partial^{2} E}{\partial z^{2}}=-\frac{1}{\epsilon_{0}} \frac{\partial^{2} p}{\partial t^{2}}
$$

where $2 K$ is the rate of decay of energy in the laser mode due to mirror reflectivities or distributed losses within the cavity. For the polarization $p(z, t)$ we have

$$
\begin{aligned}
p(z, t)= & \frac{1}{\Delta v} \sum_{\mu} \operatorname{ex}_{12}\left(\rho_{12}^{(\mu)}(t)+\rho_{21}^{(\mu)}(t)\right)+\sum_{\nu} \\
& \cdot \operatorname{ex}_{12}^{\prime}\left(\rho_{12}^{(\nu)}(t)+\rho_{21}^{(\nu)}(t)\right)
\end{aligned}
$$

with the summation extending over all laser atoms $\mu$ and absorber atoms $\nu$ within a small volume $\Delta v$ located at position $z$. Substituting for $E(z, t)$ from (6) into (13), summing over all volumes $\Delta v$, and combining the resulting equation with (9)(12) and (14), we arrive at an equation for the field amplitude $b(t)$ of the Van der Pol form

$$
\dot{b}(t)-b(t)\left\{\gamma^{\prime}-\beta^{\prime} b^{*} b\right\}=0 \text {. }
$$


The gain parameter $\gamma^{\prime}$ and saturation parameter $\beta^{\prime}$ are defined by

$$
\begin{aligned}
\gamma^{\prime} & \equiv \frac{g^{2} \sigma_{0}}{\gamma_{2}}-\frac{g_{1}^{2} N_{A}}{\gamma_{2}^{\prime}}-K \\
\beta^{\prime} & \equiv \frac{4 g^{4} \sigma_{0}}{\gamma_{1} \gamma_{2}^{2}}-\frac{4 g_{1}^{2} N_{A}}{\gamma_{1}^{\prime} \gamma_{2}^{\prime 2}}
\end{aligned}
$$

with the coupling constants $g$ and $g_{1}$ given by

$$
\begin{gathered}
g \equiv \sqrt{\frac{e^{2} x_{12}^{2} \omega_{0}}{2 \epsilon_{0} \hbar V}} \\
g_{1} \equiv \sqrt{\frac{e^{2} x_{12}^{\prime 2} \omega_{0}}{2 \epsilon_{0} \hbar V} .}
\end{gathered}
$$

Equation (15) is derived under the usual assumptions that the time variation in the net atomic inversion and the polarization amplitude is slow compared to the atomic decay times and that the laser inversion may be expanded as

$$
\frac{1}{1+\frac{4 g^{2}}{\gamma_{1} \gamma_{2}} b^{*} b} \simeq\left(1-\frac{4 g^{2}}{\gamma_{1} \gamma_{2}} b^{*} b\right)
$$

with a similar expression for the absorbing medium

$$
\frac{1}{1+\frac{4 g_{1}^{2}}{\gamma_{1}^{\prime} \gamma_{2}^{\prime}} b^{*} b} \simeq\left(1-\frac{4 g_{1}^{2}}{\gamma_{1}^{\prime} \gamma_{2}^{\prime}} b^{*} b\right) \text {. }
$$

We thus restrict our attention to the case where saturation of the absorbing species is adequately described by a first-order expansion in the laser intensity. To treat the more general case of a saturable absorber in the laser cavity, a different approach is required [17], [18] .

To account for spontaneous emission in the laser-absorber system, we supplement (15) by the addition of a noise term $\Gamma(t)$ to produce the well-known Langevin equation [14] , [19]

$$
b(t)-b(t)\left\{\gamma^{\prime}-\beta^{\prime} b^{*} b\right\}=\Gamma(t) .
$$

Here $\Gamma(t)$ is taken to be a delta-correlated Gaussian random process of zero mean representing the effect of spontaneous emission into the field mode and is such that

$$
\begin{aligned}
\left\langle\Gamma(t) \Gamma^{*}\left(t^{\prime}\right)\right\rangle & =2 q^{\prime} \delta\left(t-t^{\prime}\right) \\
\langle\Gamma(t) \Gamma(t)\rangle & =0 .
\end{aligned}
$$

The constant $q^{\prime}$ is determined by the requirement that the time rate of change of the photon number $(d / d t) b^{*} b$ derived from (21) correctly gives the contribution from spontaneous emission and is found to be

$$
q^{\prime}=\frac{2 g^{2}}{\gamma_{2}}\left\langle N_{2}\right\rangle+\frac{2 g_{1}^{2}}{\gamma_{2}^{\prime}}\left\langle N_{2}^{A}\right\rangle
$$

where $\left\langle N_{2}\right\rangle$ and $\left\langle N_{2}^{A}\right\rangle$ give the excited state populations of laser and absorber atoms, and $2 g^{2} / \gamma_{2}$ is the rate of spontaneous decay into one mode of the field from a transition of homogeneous width $\boldsymbol{\gamma}_{2}$.

As can be seen from (21), the problem of laser plus absorber atoms has now been expressed in a form identical to the usual semiclassical treatments [14] , [19] but with a modification of the constants $q^{\prime}, \beta^{\prime}, \gamma^{\prime}$ to include the presence of the absorbing medium in the laser cavity. We can therefore proceed directly from the Langevin equation (21) for the field amplitude $b(t)$ to the steady-state solution for the probability distribution $P(\widetilde{I})$ of the intracavity laser light intensity [14]

$$
P(\tilde{I})=p_{0} e^{-\left[(I-a)^{2} / 4\right]}
$$

with normalization constant $p_{0}$. The laser pump parameter $a$ is defined by

$$
a \equiv \frac{\gamma^{\prime}}{\sqrt{q^{\prime} \beta^{\prime}}}
$$

and the dimensionless intensity $\tilde{I}$ is related to the intensity $I=b^{*} b$ in terms of photon number by

$$
\tilde{I} \equiv \sqrt{\frac{\beta^{\prime}}{q^{\prime}}} I .
$$

The first two moments of the light intensity $\tilde{I}$ are found from (24) to be

$$
\begin{aligned}
\langle\tilde{I}\rangle & =a+\frac{2 e-\left(\frac{a^{2}}{4}\right)}{\sqrt{\pi}\left[1+\operatorname{erf}\left(\frac{a}{2}\right)\right]} \\
\left\langle\left(\Delta \tilde{I}^{2}\right\rangle\right. & =2-\frac{2 a e^{-\left(a^{2} / 4\right)}}{\sqrt{\pi}\left[1+\operatorname{erf}\left(\frac{a}{2}\right)\right]}-\frac{4 e^{-\left(a^{2} / 2\right)}}{\pi\left[1+\operatorname{erf}\left(\frac{a}{2}\right)\right]^{2}} .
\end{aligned}
$$

\section{InTRACAVIty ENHANCEMENT NeAR THRESHOLD}

With the above modification of the semiclassical theory of the laser, we can now address the question of the effect of changes in the absorbing medium on the laser intensity. Toward this end we compute the derivative of the laser intensity $\langle I\rangle$ with respect to changes in the loss $k$

$$
\begin{aligned}
\frac{d}{d k}\langle I\rangle= & \frac{d}{d k}\left\{\sqrt{\frac{q^{\prime}}{\beta^{\prime}}}\langle\tilde{I}\rangle\right\}=\langle\tilde{I}\rangle \frac{d}{d k} \sqrt{\frac{q^{\prime}}{\beta^{\prime}}} \\
& +\sqrt{\frac{q^{\prime}}{\beta^{\prime}}} \frac{d a}{d k} \frac{d}{d a}\langle\tilde{I}\rangle
\end{aligned}
$$

where we have used (26) and where $k \equiv g_{1}^{2} N_{A} / \gamma_{2}^{\prime}$ is related to the attenuation coefficient $\alpha^{A}$ and absorption cross section $\sigma^{A}$ for the $1 \rightarrow 2$ transition in the absorber atoms by

$$
\begin{aligned}
2 k & =\alpha^{A} c \\
2 k & =c \rho^{A} \sigma^{A}
\end{aligned}
$$

with $\rho^{A}$ as the density of absorber atoms. In the present treatment, we are interested in the regime in which the absorbing medium represents a small perturbation to the operation of the laser. This statement implies that the losses associated with the absorber should be small compared to the other cavity losses, or that

$$
k \equiv \frac{g_{1}^{2} N_{A}}{\gamma_{2}^{\prime}}<<K
$$


and since $K \simeq\left(g^{2} \sigma_{0} / \gamma_{2}\right)$ in the region of threshold, we have

$$
\frac{g_{1}^{2} N_{A}}{\gamma_{2}^{\prime}}<<\frac{g^{2} \sigma_{0}}{\gamma_{2}} \text {. }
$$

Making use of this inequality in performing the differentiation in (29), we find that

$$
\begin{aligned}
\frac{d}{d k}\langle I\rangle \simeq & \sqrt{\frac{q^{\prime}}{\beta^{\prime}}}\left\{-\frac{1}{\sqrt{\beta^{\prime} q^{\prime}}} \frac{d}{d a}\langle\tilde{I}\rangle+\frac{1}{2} \frac{g_{1}^{2} \gamma_{1} \gamma_{2}^{2}}{\gamma_{1}^{\prime} \gamma_{2}^{\prime} \sigma_{0} g^{4}}\right. \\
& \left.\cdot\left(a \frac{d}{d a}\langle\tilde{I}\rangle+\langle\tilde{I}\rangle\right)\right\} .
\end{aligned}
$$

The second group of terms in (32) is associated with a change in the coherence time of the laser intensity fluctuations and in the mean number of photons in the laser cavity at threshold. Because we assume the absorber atoms represent a small loss [see (31)], these terms make a negligible contribution to the change in laser intensity, as can be seen by noting that near the laser threshold $(d / d a)\langle\widetilde{I}\rangle$ and $(a(d / d a)\langle\widetilde{I}\rangle+\langle\tilde{I}\rangle)$ are comparable, while the ratio

$$
\phi \equiv \frac{\frac{1}{2} \frac{g_{1}^{2}}{\gamma_{1}^{\prime} \gamma_{2}^{\prime}} \frac{\gamma_{1} \gamma_{2}^{2}}{\sigma_{0} g^{4}}}{1 / \sqrt{\beta^{\prime} q^{\prime}}} \ll 1
$$

as is shown in the Appendix. In view of these observations, we may express (32) as

$$
\frac{d}{d k}\langle I\rangle \simeq-\frac{1}{\beta^{\prime}} \frac{d}{d a}\langle\tilde{I}\rangle
$$

which together with (26) yields the following expression for the fractional change in laser intensity:

$$
\frac{\frac{d}{d k}\langle I\rangle}{\langle I\rangle}=-\frac{1}{\sqrt{\beta^{\prime} \cdot q^{\prime}}}\left\{\frac{\frac{d}{d a}\langle\tilde{I}\rangle}{\langle\tilde{I}\rangle}\right\} .
$$

The change in the laser intensity due to the absorbing medium is thus equivalent to a change brought about by varying the gain or the distributed losses of the cavity, to the extent that (20), (31), and (33) are satisfied.

At this point we note that although (35) is derived for a laser consisting of a homogeneously broadened medium in a ring cavity, the equation is generally applicable to most single-mode lasers operated near threshold. With appropriate modifications in the definitions of the laser parameters, the same equation would describe the case of a standing wave cavity and an inhomogeneously broadened medium [20] as well as offresonant operation [21].

Returning to the definitions of $k, \beta^{\prime}$, and $q^{\prime}$ [see (30), (17), and (23)], we can cast (35) into a form more directly related to experimental observations by taking one of two approaches. In the first, we use the fact that $1 / \sqrt{\beta^{\prime} q^{\prime}}$ determines the time scale for fluctuations in the laser intensity. Therefore, if some information about the correlation time $\tau_{c}$ for these intrinsic fluctuations near threshold is available, the parameter $\sqrt{\beta^{\prime} q^{\prime}}$ can be found from the relation [14]

$$
\frac{1}{\sqrt{\beta^{\prime} q^{\prime}}}=\lambda_{e} \tau_{c}
$$

where $\lambda_{e}$ is an effective decay constant derived from a solution of the Fokker-Planck equation associated with the Langevin equation (21) and tabulated in the literature [22]. Equation (35) taken with (30) and (36) gives

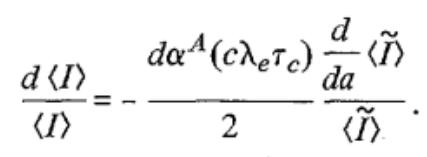

The second approach makes use of the equality

$$
\sqrt{\frac{q^{\prime}}{\beta^{\prime}}}=\eta_{0} \frac{\sqrt{\pi}}{2}
$$

which is derived from (26) and (27), with $\eta_{0}$ defined to be the mean number of photons in the cavity at threshold $(a=0)$. We have from (38)

$$
\begin{aligned}
& \frac{1}{\sqrt{\beta^{\prime} q^{\prime}}}=\frac{\sqrt{\pi}}{2} \frac{\eta_{0}}{q^{\prime}} \\
& \frac{1}{\sqrt{\beta^{\prime} q^{\prime}}} \simeq \frac{\sqrt{\pi} \eta_{0}}{K}\left(\frac{\sigma_{0}}{\left\langle N_{2}\right\rangle}\right)
\end{aligned}
$$

since $q^{\prime} \simeq \frac{1}{2} K\left(\left\langle N_{2}\right\rangle / \sigma_{0}\right)$ from (16), (23), and (31), for near threshold operation. Equation (35) can be rewritten with the help of (30) and (39) to give

$$
\frac{d\langle I\rangle}{\langle I\rangle}=-\frac{d \alpha^{A}}{\alpha} \eta_{0}\left(\frac{\sigma_{0}}{\left\langle N_{2}\right\rangle}\right) \sqrt{\pi}\left\{\frac{\frac{d}{d a}\langle\tilde{I}\rangle}{\langle\tilde{I}\rangle}\right\}
$$

where we have expressed the decay constant $2 K / c$ for the photon number in terms of an equivalent attenuation coefficient per unit length $\alpha$ representing the laser cavity losses.

Equations (35), (37), and (40) represent an extension of (3) to include the threshold region of laser operation. Far above threshold, these equations reproduce the result of (3), as can be shown from (40), for example, by noting that

$$
\frac{\frac{d\langle\tilde{I}\rangle}{d a}}{\langle\tilde{I}\rangle} \underset{a>>0}{\longrightarrow} \frac{1}{a}=\frac{1}{\eta_{0}\left(\frac{\sigma_{0}}{\left\langle N_{2}\right\rangle}\right) \sqrt{\pi}(n-1)}
$$

so that from (40) we have

$$
\frac{d\langle I\rangle}{\langle I\rangle} \underset{a>>0}{\longrightarrow}-\frac{d \alpha^{A}}{\alpha} \frac{1}{(n-1)}
$$

in agreement with (3) for operation such that $a \gg 0, n \simeq 1$. However, in contrast to (3), the fractional change in laser intensity in the region of threshold given by (40) does not become arbitrarily large but rather reaches a maximum value and then decreases, as illustrated in Fig. 2. Furthermore, the magnitude of this fractional change is dependent on the particular laser system being considered, as is evident from (35), (37), and (40). Contrary to the prediction of (3), these equations suggest that two lasers with different geometries and laser 


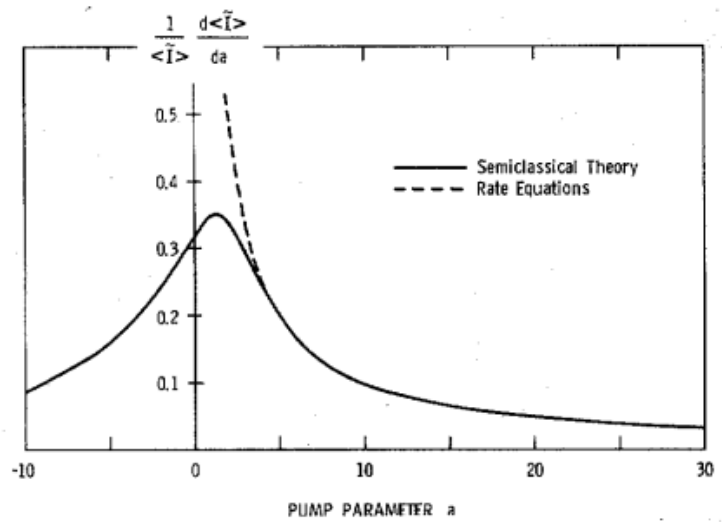

Fig. 2. Normalized change $(d\langle\tilde{I}\rangle|d a|\langle\tilde{I}\rangle$ in the dimensionless laser intensity $\langle\tilde{I}\rangle$ as a function of the pump parameter a from (40) (solid curve). The dashed curve indicates the corresponding result from (3).

media but with equal cavity losses and pumping rates will not experience the same fractional change in intensity for a change $d \alpha^{A}$ in the intracavity absorber in the region of the laser threshold. This result is a consequence of the fact that in the region of threshold the parameter $\eta_{0}$ enters the equations for the intracavity intensity change.

Finally, we note that the change in laser intensity $d\langle I\rangle$ as expressed by (35) is not independent of the working point of the laser for $a \sim 0$. However, we find that for $a \sim 5$

$$
d I=-d \alpha^{A} \cdot(\text { constant })
$$

independent of the pump parameter $a$, so that changes in laser pumping should not affect the absolute sensitivity of absorption measurements far above threshold $(a \gg 1, n \simeq 1)$ as has previously been noted [23] .

The enhancement $\xi$ defined by (4) for a single-mode laser operated in the threshold region can be obtained from (40)

$$
\frac{\Delta\langle I\rangle}{\langle I\rangle}=\left(\Delta \alpha^{A} \cdot d\right)\left\{\frac{\sqrt{\pi}}{\alpha d} \eta_{0} \frac{\sigma_{0}}{\left\langle N_{2}\right\rangle} \cdot \frac{1}{\langle\widetilde{I}\rangle} \frac{d\langle\tilde{I}\rangle}{d a}\right\}
$$

so that

$$
\xi=\frac{\sqrt{\pi}}{\alpha d} \eta_{0} \frac{\sigma_{0}}{\left\langle N_{2}\right\rangle}\left\{\frac{1}{\langle\tilde{I}\rangle} \frac{d\langle\tilde{I}\rangle}{d a}\right\}
$$

with $d \equiv$ cavity length. The loss associated with the absorber is given by $\left(\Delta \alpha^{A} \cdot d\right)$. Should the absorbing medium fill only a fraction of the cavity, as in Fig. 1, this loss would be given by $\left(\Delta \alpha^{T} \cdot d_{1}\right)$, with $d_{1} \equiv$ length of the absorption cell.

An estimate of the magnitude of $\xi$ can be made by noting that the loss $\alpha$ in a cavity, exclusive of the absorber, is

$$
\alpha=\alpha_{D}-\frac{1}{d} \ln R
$$

where $\alpha_{D}$ represents the distributed losses in the cavity and $R$ gives the reflectivity of the cavity mirrors. If the cavity losses are dominated by the loss through the mirrors, we may write

$$
\alpha \simeq \frac{(1-R)}{d} .
$$

The value of the photon number at threshold varies with laser geometry [24] and with the laser material, but reasonable

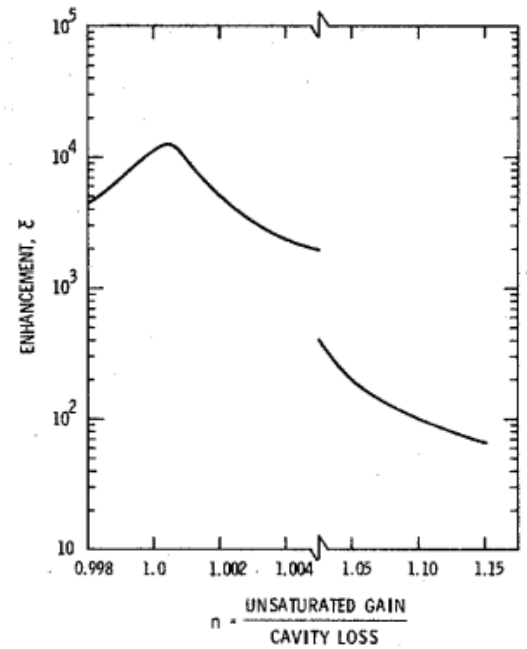

Fig. 3. Intracavity enhancement $\xi$ calculated from (44) for the parameters $\eta_{0}=2000, R=0.9$, and $\sigma_{0} /\left\langle N_{2}\right\rangle=1$. The abscissa gives the laser working point and is related to the pump parameter a by $n=1+$ $\left(a / \sqrt{\pi} n_{0}\right)\left(\left\langle N_{2}\right\rangle / \sigma_{0}\right)$.

values for $\eta_{0}$ suitable for many lasers are on the order of several thousand [25], [26]. For $\eta_{0}=2 \times 10^{3}$ and $R=0.9$, we calculate the enhancement $\xi$ from (44). Fig. 3 shows that it reaches a maximum value of approximately $10^{4}$. Alternatively, we may use (37) with coherence time $\tau_{c}=10 \mu \mathrm{s}$ and with $d=l \mathrm{~m}-\mathrm{a}$ choice of parameters that is appropriate for certain single mode dye lasers [27] -to find that $\xi_{\max } \sim 3 \times 10^{3}$ in the threshold region.

In terms of detection sensitivity for trace quantities of an absorbing material inside the laser cavity, the present treatment sets a limit on the minimum detectable $\operatorname{loss} L_{\min }$ given by

$$
L_{\min } \equiv\left(\Delta \alpha^{A} d_{1}\right)_{\min }=\left(\frac{\Delta\langle I\rangle}{\langle I\rangle}\right)_{\min }\left(\frac{1}{\xi}\right)
$$

with $\left(\Delta\langle I\rangle\langle\langle I\rangle)_{\min }\right.$ equal to the smallest fractional change in laser intensity that can be accurately sensed within the measurement interval. This change in laser intensity might be brought about by Stark or Zeeman tuning of the absorption line into resonance with the laser frequency [7] or by modulation of the laser frequency. For the choices $(\Delta\langle I\rangle /\langle I\rangle)=-0.01$ and $\xi=10^{4}$, we find

$$
L=10^{-6}
$$

so that for a $10 \mathrm{~cm}$ absorption cell

$$
\left(\Delta \alpha^{A}\right)_{\min }=10^{-7} \mathrm{~cm}^{-1} \text {. }
$$

This detection limit will vary with the particular laser under consideration but, in general, a reduction in the cavity losses (or equivalently the threshold inversion) will produce a corresponding decrease in the minimum detectable concentration through an increase in the enhancement.

Of course, since the intensity of a laser operated near its threshold exhibits large fluctuations on a microsecond time scale [27]-[29], the duration of intracavity absorption measurements for a $\mathrm{CW}$ single-mode laser operating near threshold must be sufficiently long so that these intrinsic fluctuations average to a level below the absorption signal being sought. Thus, the sacrifice for the increased sensitivity obtained by 
operating near threshold is a reduced time response capability for the system. However, since the time $T$ required to reduce the uncertainty in $\langle I\rangle$ to a level of $\delta\langle I\rangle$ is expected to be

$$
T \sim \frac{1}{\delta^{2}} \frac{1}{\sqrt{\beta^{\prime} q^{\prime}}} \frac{1}{a^{3}}, \quad a \sim 5
$$

where $\sqrt{\beta^{\prime} q^{\prime}}$ is related to the laser coherence time $\tau_{c}$ by (36), and since the enhancement $\xi$ of the system decreases only as $1 / a$ for $a \widetilde{>}$, a modest decrease in the enhancement results in a significant improvement in the time response of the system. For example, for the dye laser discussed by Abate et al. [27], $1 / \sqrt{\beta^{\prime} q^{\prime}} \sim 50 \mu \mathrm{s}$, so that for $\delta=5 \times 10^{-4}, T(a=5) \sim 1.5 \mathrm{~s}$ and $T(a=10) \sim 0.2 \mathrm{~s}$, producing a sevenfold reduction in the time required for the measurement, while the enhancement is reduced only by a factor of 2 .

\section{Discussion}

We have presented an expression for the effect of an intracavity absorber on the intensity of a single-mode $\mathrm{CW}$ laser operated near the laser threshold. The calculation shows that the range of validity of the simple expression (3) extends to very near threshold, $a \gtrsim 5$, corresponding to $(n-1) \approx 2 \times 10^{-3}$ for the case illustrated in Fig. 3. Except in a very small region near threshold, the enhancement in laser intracavity absorption spectroscopy is set by the passive cavity losses (e.g., mirror reflectivities) and not by the contribution from spontaneous emission. However, care must be taken to consider the intrinsic fluctuations of the laser intensity for small values of the pump parameter when considering the time scale for the measurements. Enhancements in detection sensitivity over conventional single pass absorption spectroscopy of order $10^{4}$ are predicted for a single-mode $\mathrm{CW}$ laser.

\section{APPENDIX}

We wish to examine the ratio

$$
\phi \equiv \frac{\frac{1}{2} \frac{g_{1}^{2}}{\gamma_{1}^{\prime} \gamma_{2}^{\prime}} \frac{\gamma_{1} \gamma_{2}^{2}}{\sigma_{0} g^{4}}}{1 / \sqrt{\beta^{\prime} q^{\prime}}} .
$$

Substituting from (17) and (23) for $\beta^{\prime}$ and $q^{\prime}$, and with the inequality (21), we find that

$$
\phi \cong \frac{2 g_{1}^{2}}{\gamma_{2}^{\prime} \gamma_{1}^{\prime}} \sqrt{\frac{\gamma_{1} \gamma_{2}}{2 g^{2}} \frac{\left\langle N_{2}\right\rangle}{\sigma_{0}}} .
$$

Now, $2 g^{2} / \gamma_{2}$ gives the rate of spontaneous emission into the laser mode by a laser atom. The ratio of this rate to the total spontaneous emission rate $W$ into all modes of the cavity that lie under the homogenous linewidth is

$$
\frac{\left(\frac{2 g^{2}}{\gamma_{2}}\right)}{W}=\frac{2}{\pi P}
$$

where $P$ is defined to be the number of cavity modes within the homogeneous line. Likewise, we find for the absorber atoms

$$
\left(\frac{2 g_{1}^{2}}{\gamma_{2}^{\prime}}\right) / W^{\prime}=\frac{2}{\pi P^{\prime}}
$$

so that

$$
\phi=\sqrt{\frac{2}{\pi} \frac{W^{\prime} \gamma_{1}\left\langle N_{2}\right\rangle}{W \gamma_{1}^{\prime} \sigma_{0}}} \frac{\sqrt{P}}{P^{\prime}} .
$$

Assuming that the gain widths and spontaneous emission rates for laser and absorber atoms are comparable gives

$$
\phi \approx \sqrt{\frac{2}{\pi}} \sqrt{\frac{\left\langle N_{2}\right\rangle}{\sigma_{0}}} \frac{1}{\sqrt{P}}
$$

since $\mathrm{W}<\gamma_{1}$. The value of $P$ is dependent upon the particular laser system considered, but a conservative estimate is $P \sim 10^{6}$ [25], so that

$$
\phi<10^{-3} \sqrt{\frac{\left\langle N_{2}\right\rangle}{\sigma_{0}}}
$$

Most lasers operate with a very high loss rate from the lower state, allowing us to estimate $\sqrt{\left\langle N_{2}\right\rangle / \sigma_{0}} \sim \sqrt{\left\langle N_{2}\right\rangle / N_{2}} \sim 1$. Hence, $\phi<10^{-3}$, as was stated in (33).

\section{REFERENCES}

[1] L. A. Pakhomycheva, E. A. Sviridenkov, A. F. Suchov, L. V. Titova, and S. S. Churilov, "Line structure of generation spectra of lasers with inhomogeneous broadening of the amplification line," Sov. Phys. JETP, vol. 12, pp. 43-45, 1970.

[2] N. C. Peterson, M. J. Kurylo, W. Braun, A. M. Bass, and R. A. Keller, "Enhancement of absorption spectra by dye-laser quenching," J. Opt. Soc. Amer., vol. 61, pp. 746-750, June 1971.

[3] R. A. Keller, E. F. Zalewski, and N. C. Peterson, "Enhancement of absorption spectra by dye-laser quenching, II," J. Opt. Soc. Amer., vol. 62, pp. 319-326, Mar. 1972.

[4] R. A. Keller, J. D. Simmons, and D. A. Jennings, "Enhancement of absorption spectra by dye-laser quenching, III: Quantitative aspects and a comparison of flash-lamp-pumped and cw systems under high resolution," Jo Opt. Soc. Amer, vol. 63, pp. 15521555, Dec. 1973.

[5] V. M. Baev, T. P. Belikova, E. A. Sviridenkov, and A. F. Suchkov, "Intracavity laser spectroscopy with continuously and quaiscontinuously operating lasers," Sov. Phys. JETP, vol. 47, pp. 2129, Jan. 1978.

[6] G. H. Atkinson, A. H. Laufer, and M. J. Kurylo, "Detection of free radicals by an intracavity dye laser technique," J. Chem. Phys., vol. 59, pp. 350-354, July 1973.

[7] J. M. Cook, K. M. Evenson, C. J. Howard, and R. F. Curl, Jr., "Laser magnetic resonance spectrum of $\mathrm{HCO}$ on the $\mathrm{D}_{2} \mathrm{O} 108 \mu$ laser line," J. Chem. Phys., vol. 64, pp. 1381-1388, Feb. 1976.

[8] R. G. Bray, W. Henke, S. K. Liu, K. V. Reddy, and M. J. Berry, "Measurement of highly forbidden optical transitions by intracavity cw dye laser spectroscopy," Chem. Phys. Lett., vol. 47, pp. 213-218, Apr. 1977.

[9] T. W. Han̈sch, A. L. Schawlow, and P. E. Toschek, "Ultrasensitive response of a cw dye laser to selective extinction," IEEE J. Quantum Electron., vol. QE-8, pp. 802-804, Oct. 1972.

[10] W. Brunner and H. Paul, "On the theory of selective intracavity absorption," Opt. Commun., vol. 12, pp. 252-255, Nov. 1974.

[11] W. Brunner and H. Paul, "Coexistence and noncoexistence of modes in a multi-mode laser," Opt. Commun., vol. 24, pp. 16-20, Jan. 1978.

[12] H. K. Holt, "Laser intracavity absorption," Phys. Rev. A, vol. 11, pp. 625-629, Feb. 1975.

[13] - "Laser intracavity absorption," Phys. Rev. A, vol. 14, pp. 1901-1902, Nov. 1976.

[14] H. Risken, "Statistical properties of laser light," in Progress in Optics, vol. VIII. Amsterdam, The Netherlands: North Holland, 1970, pp. 241-294.

[15] A. Yariv, Quantum Electronics. New York: Wiley, 1975, ch. 7 and 9.

[16] N. Dutta, R. T. Warner, and G. J. Wolga, "Sensitivity enhancement of a spin-flip Raman laser absorption spectrometer through use of an intracavity absorption cell," Opt. Lett., vol. 1, pp. 156157, Nov. 1977.

[17] R. Salomaa and S. Stenholm, "Observable manifestations of phase transitions in lasers," Appl. Phys., vol. 14, pp. 355-360, 1977. 
[18] L. A. Lugiato, P. Mandel; S. T. Dembinski, and A. Kossakowski, "Semiclassical and quantum theories of bistability in lasers containing saturable absorbers," Phys. Rev. A, vol. 18, p. 238, 1978.

[19] R. D. Hempstead and M. Lax, "Classical noise. VI. Noise in selfsustained oscillators near threshold," Phys. Rev., vol. 161, pp. 350-366, Sept. 1967.

[20] V. Arzt, H. Haken, H. Risken, H. Sauermann, C. Schmid, and W. Weidlich, "Quantum theory of noise in gas and solid state lasers with an inhomogenously broadened line. I," Z. Physik, vol. 197, pp. 207-227, 1966.

[21] H. Risken and H. D. Vollmer, "The transient solution of the laser Fokker-Planck equation," Z. Physik, vol. 204, pp. 240-253, 1967.

[22] H. Risken, "Zur statistik des laser lichts," Fortschs. Physik, vol. 16, pp. 261-308, 1968.

[23] C. J. Howard and K. M. Evenson, "Laser magnetic resonance study of the gas phase reactions of $\mathrm{OH}$ with $\mathrm{CO}$, $\mathrm{NO}$, and $\mathrm{NO}_{2}$," J. Chem. Phys., vol. 61, pp. 1943-1952, Sept. 1974.

[24] C.-Y. Huang and L. Mandel, "Effect of optical-cavity length on laser photon statistics," Phys. Rev. A, vol. 18, pp. 644-654, Aug. 1978.

[25] A. E. Siegman, An Introduction to Lasers and Masers. New York: McGraw-Hill, 1971, ch. 10.

[26] D. E. McCumber, "Intensity fluctuations in the output of $\mathrm{cw}$ laser oscillators. I," Phys. Rev., vol. 141, pp. 306-322, Jan. 1966.

[27] J. A. Abate, H. J. Kimble, and L. Mandel, "Photon statistics of a dye laser," Phys. Rev. A, vol. 14, pp. 788-795, Aug. 1976.
[28] A. W. Smith and J. A. Armstrong, "Laser photon counting distributions near threshold," Phys. Rev. Lett., vol. 16, pp. 11691172 , June 1966.

[29] S. Chopra and L. Mandel, "Correlation function of a laser beam near threshold," in Coherence and Quantum Optics. New York: Plenum, 1973, pp. 805-813.

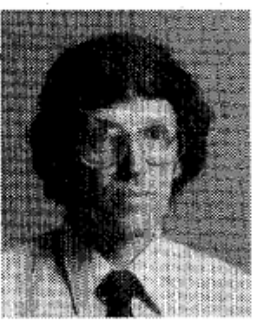

H. J. Kimble was born in Floydada, TX, on April 23, 1949. He received the B.S. degree from Abilene Christian University, Abilene, TX, in 1971, and the M.A. and Ph.D. degrees in 1973 and 1978, respectively, from the University of Rochester, Rochester, NY, all in physics.

At Rochester he was involved in investigations of resonance fluorescence. From 1977 to 1979 he was with the Physics Department of General Motors Research Laboratories, Warren, MI, where his research dealt with the characterization of lead-salt injection lasers and applications of these devices in high resolution spectroscopy. $\mathrm{He}$ is currently an Assistant Professor of Physics at the University of Texas, Austin.

Dr. Kimble is a member of the American Physical Society and the Optical Society of America.

\title{
Exact Solution of the Rate Equations for the Three Energy Level Laser
}

\author{
EZIO MARCHI, LEOPOLDO MILLÁN, AND EDGAR CRINÓ
}

\begin{abstract}
The system of two nonlinear differential equations for a three-level laser is solved exactly. It relates the number of photons $q(t)$ in the laser cavity and the population inversion $n(t)$.

The functions $n(t)$ and $q(t)$ are expanded in a power series and two recurrence relations are obtained. These are solved and the solutions are in excellent agreement with experimental results.
\end{abstract}

\section{INTRODUCTION}

W E considered a laser medium inside a resonant cavity with high reflectance mirrors and subjected to an excitation pump. We suppose that the intensity field and the population difference $n(t)=n_{2}(t)-n_{1}(t)$ are uniformly distributed in the cavity. Under these conditions, the time variation of the populations $n_{2}(t)$ and $n_{1}(t)$ are related by the equation

$$
\frac{d n}{d t}=\frac{d n_{2}}{d t}-\frac{d n_{1}}{d t}=W-2 B n q-B p_{0} n
$$

Manuscript received August 15, 1979.

The authors are with the Department de Fiscia, Universidad Nacional de San Luis, 5700, San Luis, Argentina. where $n_{2}(t)$ and $n_{1}(t)$ are the number of atoms or molecules excited to the upper and lower level in the resonator, respectively; $q(t)$ is the field intensity in the resonator measured in units of $h \nu ; t_{c}$ is the characteristic decay time of the cavity; $B$ is Einstein's coefficient; $W(t)$ is the pumping function defined as the pumping rate exceeding the pump rate necessary for equal population; and $p_{0}$ is the total number of modes.

The time variation of the field intensity in the resonator is related with the balance between the spontaneous emission in $p$ active modes, the stimulated emission, and the resonator losses by the equation

$$
\frac{d q}{d t}=B n q+\frac{1}{2} B p n+\frac{1}{2} B N_{0} p-\frac{q}{t_{c}}
$$

where $N_{0}(t)=n_{1}(t)+n_{2}(t)$ is the total number of atoms or molecules in the upper and lower laser levels.

Equations (1) and (2) are the rate equations for the threelevel laser and up to now do not have an exact analytical solution. In fact, they have been solved only in approximate form under steady-state conditions (in which case they became a set of algebraic equations), or when the departure from steady 